\title{
Hepatic Necrosis, CTCAE 5.0
}

National Cancer Institute

\section{Source}

National Cancer Institute. Hepatic Necrosis, CT CAE 5.0. NCI Thesaurus. Code C146736.

A disorder characterized by a necrotic process occurring in the hepatic parenchyma. 